\title{
Prevalence of medication
}

\section{management by community pharmacists in patients of a Newfoundland family medicine clinic}

\author{
Carla M. Dillon, BScPharm, ACPR, PharmD; John J. Mahoney; Terri L. Genge, BScBiochem, \\ BScPharm, PharmD; Amy E. Conway, BScPharm, BEd, PharmD; Katherine C. Stringer,
} $M B C h B, C C F P$

\section{Introduction}

Although the degree to which pharmacists may renew or adapt prescriptions varies widely by province, the overarching trend of expanded services is clearly growing. ${ }^{1-3}$ With the addition of reimbursement programs in some provinces ${ }^{1}$ and the introduction of prescriptive authority in oth$\mathrm{ers}^{2,4}$ there is clear growth in pharmacists' scope of practice. As a result, pharmacists have more responsibility for and input into patient care.

Medication management is an umbrella term that encompasses a variety of professional activities undertaken by a registered pharmacist to optimize safe and effective drug therapy outcomes for patients. ${ }^{5,6}$ Currently in Newfoundland and Labrador (NL), medication management includes providing an interim supply of medications, extending a prescription and adapting a prescription. ${ }^{6}$ The majority of Canadian pharmacists have the authority to provide these services; however, there are differences between provinces in what is permitted and how this authority is attained. ${ }^{7}$ In NL, both providing an interim supply and extending a prescription entail dispensing additional medication for a previously prescribed chronic therapy. When an interim supply is provided, the original prescription may have been filled at another pharmacy. An interim supply allows for a small quantity to be given that is usually less than one refill, to bridge the time needed for the patient to see his or her prescriber or to return to his or her usual pharmacy. When a prescription is extended, an additional refill of a 90-day supply or less is given for a medication previously filled at that pharmacy. Adapting a prescription includes changing the dosage form, regimen or quantity, filling in missing information and making a nonformulary generic substitution. These medication management services cannot be applied to a narcotic, controlled drug or targeted substance, including benzodiazepines. ${ }^{6}$ In 2010, changes to the NL Pharmacy Regulations permitted the use of medication management under the procedure outlined in the NL Pharmacy Board (NLPB) Standards of Pharmacy Practice. ${ }^{6,8}$ To provide this service, NL pharmacists must provide NLPB with a signed declaration indicating they have thoroughly read and understand the medication management standard of practice. As part of the fundamental requirements for performing medication management, NLPB states that in most instances the original prescriber or the patient's primary health provider must be notified, preferably via faxing a standard form. ${ }^{6}$ In June 2012, the NL Prescription Drug Program (NLPDP) agreed to pay pharmacists for medication management services provided to NLPDP beneficiaries. ${ }^{9}$

The Ross Family Medicine Centre (RFMC) is an academic clinic in St. John's, NL. Prescribers in this clinic include 5 family physicians, 1 nurse practitioner and family medicine residents. Although the RFMC provides care to patients of all ages, this clinic specializes in geriatric care. Medication management documentation received by the RFMC is scanned into patients' electronic medical records. On January 28, 2013, the RFMC implemented a policy of referring 
patients to their community pharmacist for an interim supply or prescription extension if they ran out of medications prior to being able to see their prescriber. The change in policy was driven by the belief that this service would help to more effectively use prescribers' time by reducing time addressing calls or faxes for medication refills, while maintaining continuity of treatment for patients.

Currently, there are limited Canadian data on the use of interim supply and extending and/or adapting a prescription, and there is no information on these practices in NL. In the first year (2009) that this scope was in effect in British Columbia (BC), only $0.17 \%$ of prescriptions were renewed or adapted. Of those, $80 \%$ were prescription renewals. ${ }^{10}$ In contrast, when medication management labour costs among $10 \mathrm{BC}$ pharmacists who were known high users of medication management were studied, adaptation or renewal was applied to $8.2 \%$ prescriptions over a 40-hour work period. Of those, only $47 \%$ involved renewing a prescription..$^{11}$ Given the potential for optimization of patient care, a better understanding of the local prevalence of medication management use, including the frequency of specific medication management services, may lead to improvements in the process for pharmacists, prescribers and patients.

The objectives of this study were to determine the prevalence of medication management use by NL community pharmacists in patients of the RFMC and to determine the frequency of the subcategories of medication management in this same population.

\section{Methods}

In this retrospective chart review, relevant patient charts were identified by performing a search of the RFMC electronic medical records (Wolf Medical Systems, Wolf Medical Suite version 2010.1D). Medication management documentation forms in patient charts were identified by searching for documents containing one of the following in its title: medication management (all words), med management (all words), med manag (all words), med services (all words), pharm board (all words), NLPB (any words), med notification (all words), interim (any words), extension (any words), adaptation (any words), pharmacy (any words), pharmacist (any words), from pharm (all words), from drug (all words), fax pharm (all words) or fax drug (all words). The search was further refined by restricting it to active patients of the RFMC of any age. The search was limited to the 1-year period starting July 10,2012, and ending July 11,2013 . This time frame reflected a period when pharmacists were believed to be familiar with medication management, as it had been in place since 2010, and a period where use may have been increasing, due to the start of reimbursement by NLPDP in the month prior. This period also encompassed the January 2013 change in policy, mentioned above, regarding prescription extension at RFMC. To determine the total population of the RFMC, a clinic patient list was generated on July 11, 2013, by searching for all active patients of the RFMC of any age.

Upon identification of medication management documentation forms, the following data were gathered using a data collection form: patient demographics, pharmacist registration year, type of medication management provided, reason for providing medication management services and the medication in question.

Results were presented in a descriptive manner using frequencies, averages, medians and standard deviations as appropriate. Ethics approval was received from the Health Research Ethics Authority of NL.

\section{Results}

A total of 110 medication management services were provided to 82 patients within the time frame ( 1 year), which resulted in a prevalence of medication management in the RFMC population of $4.6 \%$. Those who received medication management tended to be older than the overall clinic population (Table 1). The average number of services received per patient was $1.34 \pm$ 1.24 (range $1-11$, median $=1$ ). The majority of patients $(n=69,84 \%)$ received medication management on a single occasion.

Breaking down the services provided showed that $56 \%$ were prescription extensions, $43 \%$ provided an interim supply and $<1 \%$ were prescription adaptations (Table 2). It was noted that medication management for multiple medications was documented in 2 different ways. In some cases, a separate medication management form was completed for each medication, while in other cases a single medication management form was used with multiple prescription labels attached.

Medication management was provided for a broad range of medications (Table 3 ). Of the 
TABLE 1 Patient characteristics

\begin{tabular}{l|c|c}
\hline & MM patients & Total clinic population \\
\hline$n$ & 82 & 1802 \\
\hline Female gender, $\%$ & 51 & 52 \\
\hline Mean \pm SD age, years & $67 \pm 18.8$ & $48 \pm 24.1$ \\
\hline Median age, years & 73 & 50 \\
\hline Age range, years & $18-94$ & $0-100$ \\
\hline
\end{tabular}

MM, medication management; SD, standard deviation.

TABLE 2 Breakdown of medication management type

\begin{tabular}{l|c|c|c}
\hline & $\begin{array}{c}\text { Extended } \\
\text { prescription }\end{array}$ & Interim supply & Adaptation \\
\hline$n$ & 62 & 47 & $\begin{array}{c}1 \text { (change in } \\
\text { quantity) }\end{array}$ \\
\hline Reason: No refills, $n$ & 51 & 33 & NA \\
\hline Reason: Prescription expired, $n$ & 4 & 3 & NA \\
\hline Reason: Other or not available, $n$ & 7 & 11 & $\begin{array}{c}\text { Reimbursement } \\
\text { and package size }\end{array}$ \\
\hline Mean \pm SD length of therapy, days & $24.58 \pm 9.64$ & $9.38 \pm 4.84$ & NA \\
\hline Median length of therapy, days & 30 & 7 & NA \\
\hline Range, days & $3-45$ & $2-30$ & \\
\hline
\end{tabular}

NA, not applicable; SD, standard deviation.

113 medications involved, the most common class of medications was cardiovascular (35\%), followed by central nervous system (17\%) and diabetes medications or supplies (13\%).

The services were provided by 60 pharmacists, with the majority of medication management ( $n=61,55 \%)$ completed by pharmacists registered in 2008 or later. An average of $1.83 \pm 2.04$ services were completed per pharmacist, with a median of 1 and a range of 1 to 12 per pharmacist.

After the January 2013 change in policy at the RFMC, the number of medication management services increased from an average of 8.6 per 30 days to an average of 9.5 per 30 days.

\section{Discussion}

In this retrospective study of patients of an academic family medicine clinic, medication management services were received by approximately 1 in 22 patients or $4.6 \%$ over 1 year.
The most common subcategory of medication management provided was prescription extension at $56 \%$, closely followed by interim supply, whereas adaptation was rare. For both extension and interim supply, lack of refills was the most common reason associated with providing medication management. Those receiving the service tended to be older than the clinic population, and pharmacists providing the services tended to be more recently licensed NL pharmacists. The clinic's change in policy to no longer calling in refills and to referring patients to their pharmacy for an interim medication supply was associated with a slight increase in the number of medication management services performed and may result in a medication management prevalence that is higher than in the greater St. John's region.

Consistent with Marra and colleagues' ${ }^{10}$ findings in $\mathrm{BC}$, this study found that the majority of medication management services provided involved supplying additional medication. This 
TABLE 3 Type of medications

\begin{tabular}{l|c|l}
\hline & $n$ & \multicolumn{1}{|c}{ Most common medications } \\
\hline Cardiovascular & 39 & $\beta$-blocker $(n=9)$, ACEI $(n=8)$, ARB $(n=5)$, statin $(n=5)$ \\
\hline Central nervous system & 19 & TCA $(n=5)$, SNRI $(n=3)$, SSRI $(n=3)$ \\
\hline Diabetes (medications or supplies) & 15 & Supplies $(n=7)$, metformin $(n=4)$, insulin $(n=3)$ \\
\hline Levothyroxine & 9 & \\
\hline Gastrointestinal & 6 & PPI $(n=3)$, sucralfate $(n=2)$, ranitidine $(n=1)$ \\
\hline Inhaler (oral or nasal) & 5 & \\
\hline Vitamins/mineral & 5 & \\
\hline Topical & 4 & \\
\hline Analgesics & 3 & \\
\hline Erectile dysfunction & 3 & \\
\hline Contraceptive & 2 & \\
\hline Eye drops & 2 & \\
\hline Antibiotic* & 1 & \\
\hline
\end{tabular}

ACEI, angiotensin-converting enzyme inhibitor; ARB, angiotensin receptor blocker; PPI, proton pump inhibitor; SNRI, serotonin-norepinephrine receptor inhibitor; SSRI, selective serotonin receptor inhibitor; TCA, tricyclic antidepressant. *Long-term use for urinary tract infection prophylaxis.

may reflect pharmacists' comfort with this form of prescribing. Pharmacists practising in Alberta indicated in the study by Makowsky et $\mathrm{a}^{12}$ that this form of medication management legitimized the previous practice of lending a patient medication to prevent interruptions in therapy. In the present study, there was a large variation in the quantity of medication supplied for both prescription extension and interim supply. Under the NL standard, extending a prescription is intended to provide an additional refill, while an interim supply is intended to provide just enough medication until the patient can see his or her prescriber or go to his or her usual pharmacy. The practice of extending a prescription for as little as 3 days and providing an interim supply for as long as 30 days suggests there may be some misunderstanding on the part of pharmacists between these 2 similar forms of medication management. However, the appropriate application of medication management was outside the scope of this study. When an interim supply or prescription extension was needed for multiple medications at the same time, there was variation in the documentation. Some pharmacists completed a form for each medication, while others provided all of the required information on one form. The inconsistency in the application and documentation may suggest that further education or clarification is needed in the NLPB standards of practice.

Unlike the Marra et $\mathrm{a}^{10}$ findings, where adaptation (i.e., therapeutic substitutions and change in dose, regimen or formulation) accounted for $20 \%$ of medication management, only a single adaptation $(0.9 \%)$ was found in this sample. This low rate was an unexpected finding and warrants further study.

It is of interest that over half of the pharmacists (55\%) who provided medication management in this study were licensed to practice pharmacy in NL in 2008 or later. The School of Pharmacy (SoP) at Memorial University of NL changed to 1 year of general university studies plus 4 years in the SoP from 2 years of general studies plus 3 years in the SoP. Students graduating in 2008 or later have an additional year in the SoP and a revised curriculum that included a series of 7 skills courses where activities such as application of medication management would have been practised.

Patients receiving medication management in this study tended to be older than the clinic 
population. This is expected, as the number of chronic medications tends to increase with age. The high prevalence of cardiovascular medications is also reflective of an older population. Marra et $\mathrm{al}^{10}$ found that renewals were most common for ramipril, hydrochlorothiazide, levothyroxine, atorvastatin and metformin, respectively. Although there were no renewals for hydrochlorothiazide in this study, the other medications or medication classes were common.

This study looked at medication management documentation that was scanned into patients' electronic medical records (EMRs). As per the NLPB standard of practice, pharmacists could notify prescribers verbally. ${ }^{6}$ Any such communications would not have been captured by this study; however, given that faxing the documentation is standard practice, this is unlikely to have significantly affected the results. A variety of document titles were given to medication management forms in the EMR system. It is possible some forms were missed by the search terms used in this study. However, a broad range of search terms was used, which should have minimized missing relevant documents. This study occurred at a single family practice clinic, which may limit generalizability to other patient groups or pharmacists practising in other locations.

\section{Conclusion}

The prevalence of medication management use was $4.6 \%$ for this group of patients at the RFMC. The most common type of medication management used was prescription extension, for which the most common reason was lack of refills. This study adds to the very limited data regarding these types of management medication services provided by community pharmacists.

From the School of Pharmacy (Dillon, Mahoney, Genge, Conway) and Discipline of Family Medicine (Dillon, Stringer), Memorial University of Newfoundland, St. John's, Newfoundland. Contact cmdillon@mun.ca.

Funding: Provided by an unrestricted grant from the School of Pharmacy, Memorial University of Newfoundland (MUN), St. John's, NL. In-kind support by the Ross Family Medicine Clinic, Discipline of Family Medicine, $M U N$, in the form of space and computer access. The funding organization did not play a role in the design and conduct of the study, analysis and interpretation of the data, or the preparation and approval of the manuscript.

\section{References}

1. Lynas K. Saskatchewan pharmacists pleased with demand for prescribing services, but seek to expand assessments for minor ailments. Can Pharm J (Ott) 2013;146:17.

2. Lynas K. Pharmacist authority to expand in Saskatchewan and Nova Scotia. Can Pharm J (Ott) 2010;143:166.

3. Canadian Pharmacists Association. Summary of pharmacists' expanded scope of practice across Canada. January 2014. Available: www.pharmacists.ca/cpha-ca/assets/File/ pharmacy-in-canada/ExpandedScopeChart.pdf (accessed March 25, 2014).

4. Canadian Pharmacists Association. Our way forward: optimizing drug therapy outcomes for Canadians through patient-centred care. June 2013. Available: http://blueprintforpharmacy.ca/docs/pdfs/blueprint-priorities---our-wayforward-2013---june-2013.pdf (accessed September 11, 2013).

5. Canadian Pharmacists Association. Medication management definition. July 2013. Available: http://blueprintforpharmacy .ca/docs/resource-items/medication-management-definitionhandout---july-30-2013.pdf (accessed September 11, 2013).

6. Newfoundland and Labrador Pharmacy Board. Newfoundland and Labrador Pharmacy Board standards of pharmacy practice: medication management by community pharmacists. Amended April 9, 2010. Available: http://www.nlpb
.ca/media/SOPP-Medication_Management-June2010.pdf (accessed March 25, 2014).

7. Law MR, Ma T, Fisher J, Sketris IS. Independent pharmacists prescribing in Canada. Can Pharm J (Ott) 2012;145(1):17-23.e1. 8. Newfoundland and Labrador Pharmacy Board. Standards of pharmacy practice for medication management in community pharmacy approved and implemented. The Apothecary. Summer 2010. Available: http://www.nlpb.ca/media/Apothecary_ Archive_2009-2010.pdf (accessed March 25, 2014).

9. Agreement between Government of Newfoundland and Labrador and the Pharmacists' Association of Newfoundland and Labrador. June 25, 2012. Available: www.panl.net/ userfiles/files/Documents/2012/Government\%20Relations/ PANL\%20Agreement,\%202012.PDF (accessed June 27, 2013). 10. Marra C, Lynd L, Grindrod K, et al. An overview of pharmacy adaptation services in British Columbia. 2010. Available: www.health.gov.bc.ca/pharmacare/pdf/COREAdaptation Overview.pdf (accessed November 24, 2013).

11. Marra CA, Lynd LD, Grindrod KA, et al. Evaluating the labour costs associated with pharmacy adaptation services in British Columbia. Can Pharm J (Ott) 2012;145:78-82.

12. Makowsky MJ, Guirguis LM, Hughes CA, et al. Factors influencing pharmacists' adoption of prescribing: qualitative application of the diffusion of innovations theory. Implement Sci 2013;8:109. 